\title{
Path-length-resolved measurements of multiple scattered photons in static and dynamic turbid media using phase-modulated low-coherence interferometry
}

\author{
Babu Varghese \\ Vinayakrishnan Rajan \\ University of Twente \\ Biomedical Technology Institute \\ Biophysical Engineering Group \\ P. O. Box 217 \\ NL-7500AE Enschede \\ The Netherlands
}

\section{Ton G. Van Leeuwen}

University of Twente

Biomedical Technology Institute

Biophysical Engineering Group

P. O. Box 217

NL-7500AE Enschede

The Netherlands

and

Academic Medical Center

Laser Center

Miebergdreef 9

1105 AZ, Amsterdam

The Netherlands

\author{
Wiendelt Steenbergen \\ University of Twente \\ Biomedical Technology Institute \\ Biophysical Engineering Group \\ P.O. Box 217 \\ NL-7500AE Enschede \\ The Netherlands
}

\begin{abstract}
In optical Doppler measurements, the path length of the light is unknown. To facilitate quantitative measurements, we develop a phase-modulated Mach-Zehnder interferometer with separate fibers for illumination and detection. With this setup, path-length-resolved dynamic light scattering measurements of multiple scattered light in static and dynamic turbid media are performed. Optical path length distributions spanning a range from 0 to $11 \mathrm{~mm}$ are measured from the area under the phase modulation peak around the modulation frequency in the power spectrum. A Doppler-broadened phase modulation interference peak is observed that shows an increase in the average Doppler shift with optical path length, independent of absorption. Validation of the estimated path length distributions is done by measuring their deformation for increasing absorption and comparing these observations with predictions based on Lambert-Beer's law. ๑ 2007 Society of Photo-Optical Instrumentation Engineers. [DOI: 10.1117/1.2714795]

Keywords: phase modulation; Mach-Zehnder interferometer; turbid media; light; Doppler.

Paper 06177R received Jun. 30, 2006; revised manuscript received Nov. 22, 2006; accepted for publication Dec. 31, 2006; published online Mar. 15, 2007. This paper is a revision of a paper presented at the SPIE conference on Biophotonics and New Therapy Frontiers, Apr. 2006, Strasbourg, France. This paper presented there appears (unrefereed) in SPIE Proceedings Vol. 6191.
\end{abstract}

\section{Introduction}

Laser Doppler blood flowmetry is a noninvasive technique for monitoring blood microcirculation in biological tissues. The coherent light, which is delivered to the tissue through an optical fiber, interacts with static as well as moving scatterers, e.g., red blood cells. The light scattered by moving red blood cells receives a slight frequency shift due to the Doppler effect. Via a second optical fiber, some of the scattered light is guided to the detector, ${ }^{1}$ where the mixture of Doppler shifted and unshifted light causes photocurrent fluctuations. Based on the power spectrum of the photodetector signal, the conventional laser Doppler perfusion monitor records perfusion values averaged over different and basically unknown path lengths, which creates an uncertainty in the relation between the measured perfusion signal and the real perfusion. A longer path length will increase the probability that a Doppler shift will occur, thus yielding an overestimation of the blood perfusion, compared to the short path length situation. The development of techniques for monitoring Doppler shifts with

Address all correspondence to Babu Varghese, Biophysical Engineering Group, University of Twente, Hogekamp 9104, NL 7500 AE, Enschede, The Netherlands. Tel: 31534893870; FAX: 31534891105; E-mail: b.varghese@utwente.nl path length information would result in more quantitative and more reliable tissue perfusion information.

To obtain path length distributions with widths of a few millimeters, several techniques have been explored. Timeresolved measurements ${ }^{2}$ or amplitude modulation of the light intensity $^{3}$ can provide this information. However, for a spatial resolution of $50 \mu \mathrm{m}$, these techniques require either a temporal resolution of $150 \mathrm{fs}$ or electronics working in the gigahertz range. To avoid these technical challenges, coherence-gated ${ }^{4-8}$ and wavelength-modulated ${ }^{9}$ interferometric systems have been applied as an alternative approach to measure path length distributions up to ten times the scattering mean-freepath length within highly scattering media. ${ }^{4-8}$ For both the Michelson- ${ }^{4-6}$ and the Mach-Zehnder-based interferometric setups, ${ }^{7,8}$ these low-coherence interferometric measurements depended on the photons that are Doppler shifted by the Brownian motion of the particles in the medium. In contrast to most single-mode fiber optic Michelson interferometric systems used in Doppler optical coherence tomography $(\mathrm{OCT})^{10}$ which adopt on axis back reflection, we use a multimode fiber optic Mach-Zehnder interferometer, with positions for illumination and detection separated by a distance of ten to

1083-3668/2007/12(2)/024020/7/\$25.00 @ 2007 SPIE 
a hundred times the scattering mean-free-path length. This scheme offers greater flexibility, since the distance $R$ between the illumination and the detection fiber can be varied, giving one some control over the relative depth sensitivity of the system. Our measurements explore the regime of multiple scattered photons. Furthermore, a large detection window with a sufficiently small modal dispersion without affecting the resolution of the system is achieved by the use of multimode graded-index fibers in the collection of scattered light from the sample. Compared to our previous work, ${ }^{7,8}$ in this work we add the feature of phase modulation of the reference beam in the Mach-Zehnder interferometric setup. Lowcoherence interferometry with phase modulation of the reference beam has recently been adopted in single scattering spectroscopy to analyze the characteristics of extremely dense colloidal suspensions. ${ }^{11}$ The goal of this study is to demonstrate the feasibility of phase-modulated low-coherence interferometry far beyond the single scattering regime. We show that by this phase modulation, also light that has been scattered by static structures only, will contribute to the interferometric signal. Furthermore, phase modulation will enhance the signal-to-noise ratio since the signal component generated by phase modulation can be shifted to higher frequencies than the signal component caused by mutual interference of Doppler-shifted light, which occupies the low frequency range of the spectrum.

\section{Theoretical Aspects}

The fundamental output quantity of a laser Doppler perfusion monitor is the first moment of the power spectrum $P(\omega)$ of the detector signal; in general, the $i$ 'th moment is being defined as

$$
M_{i}=\int_{a}^{b} P(\omega) \omega^{i} d \omega,
$$

where $a$ and $b$ are device-dependent low and high cut-off frequencies. With $i=0$, a quantity is obtained that is proportional to the concentration of moving red blood cells, while $i=1$ describes red blood cell flux, which is the product of concentration and the root mean square of the red cell velocity, at least for low blood concentrations. ${ }^{12}$

The method presented here utilizes the values of $M_{0}$ and the Lorentzian width of the phase modulation peak appearing in the photodetector signal power spectrum to determine the path length distribution and the Doppler shift of photons as a function of optical path length, respectively. The zeroth moment $M_{0}$ of the heterodyne spectrum is the total power of the photocurrent fluctuations caused by the interference of Doppler-shifted light from the sample and reference light of fixed path length, and is proportional to the intensity of photons with a certain path length. The weighted first moment $M_{1} / M_{0}$ is equal to the average Doppler shift of the light in this path length. The power spectrum of light that is scattered by a monodisperse suspension of particles undergoing Brownian motion and is heterodyne detected is a Lorentzian distribution and the average Doppler shift, which depends on the scattering properties, and photon path length within the strongly multiple scattering medium can be obtained from the Lorentzian line width. For higher peak phase modulation

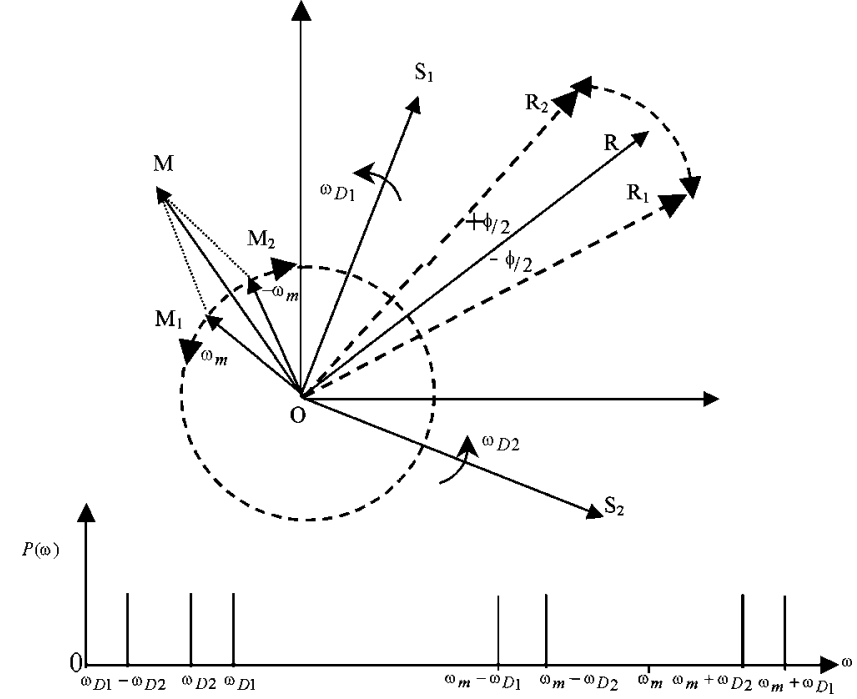

Fig. 1 Phasor diagram for the interfering fields (top) and the resulting power spectrum (bottom).

angles, the spectrum of interference signal is composed of frequency sidebands at multiples of the modulation frequency, and the path-length-dependent scattering properties of the medium can be obtained from these interference peaks. For sufficiently small phase modulation angles, higher harmonics are absent in the power spectrum, and the average Doppler shift is being obtained from the width of the resulting phase modulation peak present at the modulation frequency. This can be understood from the phasor description of the interfering fields, as shown in Fig. 1. Here the reference wave and two Doppler-shifted sample waves are represented by phasors $R$, $S_{1}$, and $S_{2}$, respectively. The angle $\phi$ is the peak phase deviation due to phase modulation in the reference light, which is depicted by a sinusoidal oscillation of the reference phasor between the extreme phasors $R_{1}$ and $R_{2}$ on either side of the average phasor $R$. For small values of $\phi$, the oscillation of the reference phasor between $R_{1}$ and $R_{2}$ is equivalent to the summation of the average reference phasor $R$, and two phasors $M_{1}$ and $M_{2}$ of equal length that rotate in opposite directions with a constant angular speed equal to the phase modulation frequency $\omega_{m}$. The initial phase of $M_{1}$ and $M_{2}$ should be chosen such that their sum phasor $M$ is perpendicular to $R$. The amplitude of $M_{1}$ and $M_{2}$ is

$$
O M_{1}=O M_{2}=\frac{1}{2} O R \tan \frac{\phi}{2}
$$

to achieve the desired phase modulation angle $\phi$. (In Fig. 1, the length of $M_{1}, M_{2}$ and $M$ is enlarged for the sake of clarity.) The sample waves $S_{1}$ and $S_{2}$ with Doppler shifts $\omega_{D 1}$ and $\omega_{D 2}$ interfere with both $M_{1}$ and $M_{2}$. Since only positive frequencies show up in the power spectrum, interference peaks are expected at $\omega_{m}-\omega_{D i}$ and $\omega_{m}+\omega_{D i}(i=1,2)$. This interference of sample light with reference light will be called "heterodyne." In practice, from a turbid sample waves are obtained with a distribution of Doppler shifts, leading to a similar distribution of spectral components centered around $\omega_{m}$. Hence the shape of the peak around $\omega_{m}$ corresponds to 
Varghese et al.: Path-length-resolved measurements...

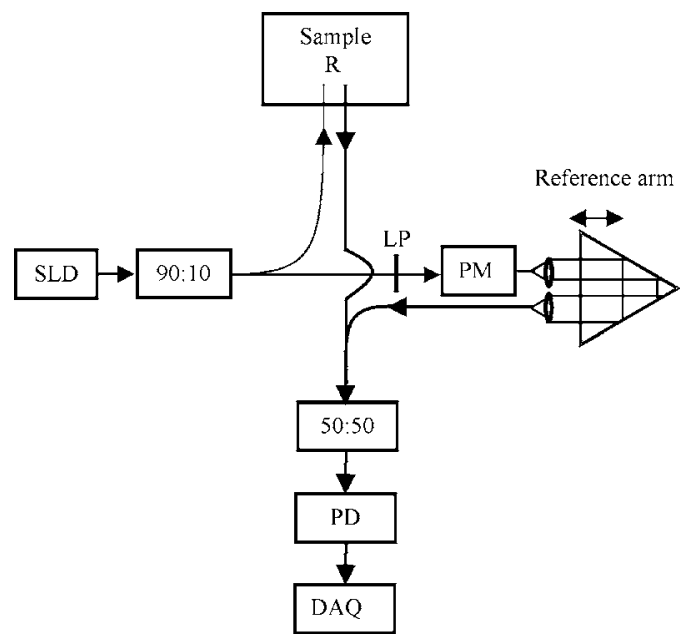

Fig. 2 Schematic of the fiber optic Mach-Zehnder interferometer. Single-mode and gradient-index multimode fibers are shown by thin and thick lines, respectively. SLD denotes superluminescent diode, PD is the photodetector, LP is a linear polarizer, PM is the electrooptic phase modulator, and 90:10 and 50:50 are single-mode and multimode fiber couplers, respectively.

the Doppler shift distribution. The component of the reference light represented by the average phasor $(O R)$ that still is at the original light source frequency will also interfere with the scattered light from the sample and thus, apart from the peak around the phase modulation frequency, a heterodyne component will also occur at low frequencies. When the peak phase modulation angle is increased, the heterodyne component in the low frequency range of the spectrum is translated to sidebands at higher harmonics. The path-length-dependent scattering properties of the medium can also be obtained from the spectrum of interference signal composed of frequency sidebands at the multiples of modulation frequency for higher peak phase modulation angles. Finally, the sample phasors $S_{1}$ and $S_{2}$ will mutually interfere to generate beats at frequency $\omega_{D 1}-\omega_{D 2}$, a component that we call "homodyne." Hence, the spectrum at low frequencies is a mixture of homodyne interference of light remitted by the sample, and heterodyne interference between sample light and unmodulated reference light, while the spectral component around the phase modulation frequency is the pure Doppler shift distribution.

\section{Materials and Methods}

We use a fiber optic Mach-Zehnder interferometer (Fig. 2) with a superluminescent diode (Inject LM2-850, $\lambda=832 \mathrm{~nm}$, $\Delta \lambda_{\mathrm{FWHM}}=17 \mathrm{~nm}$, coherence length $\left.L_{C}=18 \mu \mathrm{m}\right)$ that yields $2 \mathrm{~mW}$ of power from the single-mode pigtail fiber as the light source. A single-mode fiber optic coupler with a splitting ratio of 90:10 is used to create a reference arm $(10 \%)$ and a sample arm (90\%). Single-mode fibers (mode field diameter $=5.3 \mu \mathrm{m}, \mathrm{NA}=0.14)$ are used for illumination, while multimode graded-index fibers (core diameter $=100 \mu \mathrm{m}$, NA $=0.29$ ) are used for detection, providing a large detection window. The coherence length of the light source, and the intermodal dispersion in the detection fiber, define the path length resolution of the measurement.
The path length of the reference arm is varied by reflection of the light in a translatable retroreflector, and the position of the retroreflector is adjusted to yield an optical path length equal to the optical path length of a certain part of the photons in the sample arm. The reference beam is polarized using a linear polarizer, and the phase is sinusoidally modulated at $22 \mathrm{kHz}$ using an electro-optic broadband phase modulator (New Focus model 4002). The sinusoidal phase modulation applied to the light is set to an angle of less than $\pi / 2$ radians, so that frequency sidebands are absent in the spectra.

To validate the obtained optical path length distributions, measurements were performed on three samples with identical scattering properties but increasing absorption levels. The media were an aqueous suspension of $25 \%$ of Intralipid $20 \%{ }^{13}$ and the same suspensions with absorption coefficients of 0.50 and $0.85 \mathrm{~mm}^{-1}$. For absorption, a black dye (Royal Talens black Ecoline ${ }^{\mathrm{TM}}$ Black) was used. The transmission spectrum of a water solution of the black ink in the wavelength range 600 to $900 \mathrm{~nm}$ was measured by a spectrophotometer (Shimadzu UV-2101-PC).

The scattering medium that is used to study the ability of our method to measure on static media is based on $4 \%$ (by weight) aqueous solution of poly(vinyl alcohol) (PVA). PVA with a degree of hydrolysis greater than $99 \%$, and an average molecular weight (MW) of 85,000 to 140,000 (SigmaAldrich, catalog nr 36 314-6) was used to prepare the aqueous solutions. The desired scattering properties are obtained by adding polystyrene microspheres of $\varnothing 0.77 \mu \mathrm{m}\left(\mu_{s}^{\prime}\right.$ $\left.=2.35 \mathrm{~mm}^{-1}, g=0.85\right)$. In this form it is a highly viscous liquid. By adding 4\% (by weight) aqueous sodium borate (borax) solution as a cross-linking agent, a visco-elastic gel (slime) is formed with optical properties almost similar to those of the original viscous medium. The amount of borax solution added to the whole volume of the PVA-water-PS suspension $(12 \mathrm{~mL})$ was about $1.0 \mathrm{~mL}$ to produce a visco-elastic gel medium. Measurements obtained in these viscous and gel media were compared with those obtained in an aqueous polystyrene suspension with equal optical properties.

We measured the path length distribution for a center-tocenter fiber distance of $500 \mu \mathrm{m}$. The path length distribution is shifted for large fiber distances, which is due to the increase in the length of the shortest path from one fiber to another and is broader due to diffusive light transport. At the detector (New Focus model 2001 photoreceiver), the light that is scattered in the sample and the light from the reference arm after passing through a 50:50 fiber coupler are mixed. The ac photocurrent is measured with a 12-bit analog-to-digital converter (National Instruments), sampling at $50 \mathrm{kHz}$, the signal was sampled for $52 \mathrm{~s}$ to get an average of 1000 spectra, and is Fourier transformed. Squaring this Fourier transform yields the power spectrum of the signal. To get smooth curves, we use an average of 1000 spectra for the aqueous and viscous media, and 5000 spectra in the case of the visco-elastic medium.

The position of the retroreflector that corresponds to the zero optical path length in the medium was determined by replacing the sample by a mirror at a distance of approximately. $13 \mathrm{~mm}$ to the tips of the two fibers. From the position of the retroreflector for which a heterodyne signal was obtained, the position for a zero path length of light probing the 


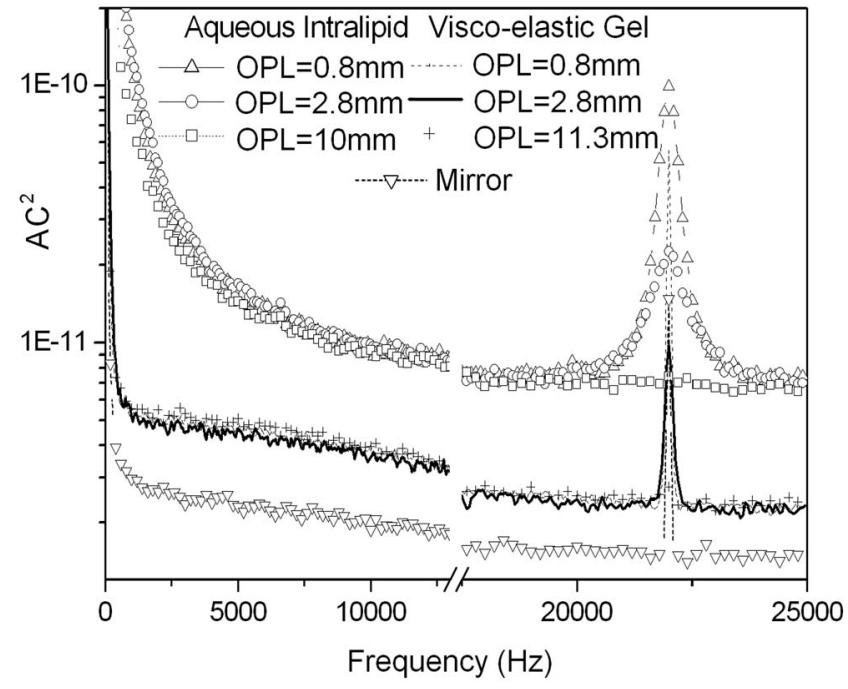

Fig. 3 Power spectra measured for three positions of the referencearm mirror, corresponding to an optical path length of $0.8,2.8$, and $11.3 \mathrm{~mm}$. An aqueous Intralipid suspension $\left(\mu_{\mathrm{a}}=0.001 \mathrm{~mm}^{-1}\right)$, a gel medium $\left(\mu_{s}^{\prime}=2.35 \mathrm{~mm}^{-1}, g=0.85\right)$, and mirror are used as the dynamic, static scattering, and reflecting media, respectively.

scattering samples could be obtained. The power spectra measured with the sample replaced by a reflecting mirror is shown in Fig. 3. The width of this spectrum is $55.34 \mathrm{~Hz}$, which can be regarded as the minimum detectable Doppler broadening in our system.

We measured spectra for a range of positions of the retroreflector in the reference arm. The path length distribution is obtained by subtraction of $M_{0}$ of the reference arm noise and of the sample arm homodyne Doppler signal from $M_{0}$ of the corresponding spectra around the modulation frequency between frequencies of 20 and $24 \mathrm{kHz}$. The ac power of the heterodyne spectra is normalized by the $\mathrm{dc}^{2}$ component to render the results independent of the power of the light source. The variation of the DC value of the reference beam for the whole path length is within $4 \%$. The modulation peak appearing in the power spectra is fitted with a Lorentzian function, and the Doppler shift is measured from the corresponding Lorentzian width of the modulation peak.

\section{Experiments and Results}

Figure 3 shows the power spectra measured for an aqueous Intralipid solution and visco-elastic gel medium with a fiber distance $(R)$ of $500 \mu \mathrm{m}$ for three positions of the retroreflector, which correspond to optical path lengths of $0.8,2.8$, and $11.3 \mathrm{~mm}$ in the sample. At optical path lengths of 0.8 and $2.8 \mathrm{~mm}$, interference peaks are observed around the phase modulation frequency of $22 \mathrm{kHz}$, while at an optical path length of $11.3 \mathrm{~mm}$ no interference is observed. For the aqueous medium, we observe the width of the phase modulation peak to increase with the optical path length. The variation that appears in the power spectra at low frequencies (0 to $10 \mathrm{kHz}$ ) measured for aqueous scattering media (Fig. 3) is the result of Doppler broadening of light scattered by particles in Brownian motion. The power spectrum, measured for widely different optical path lengths in the sample and refer-
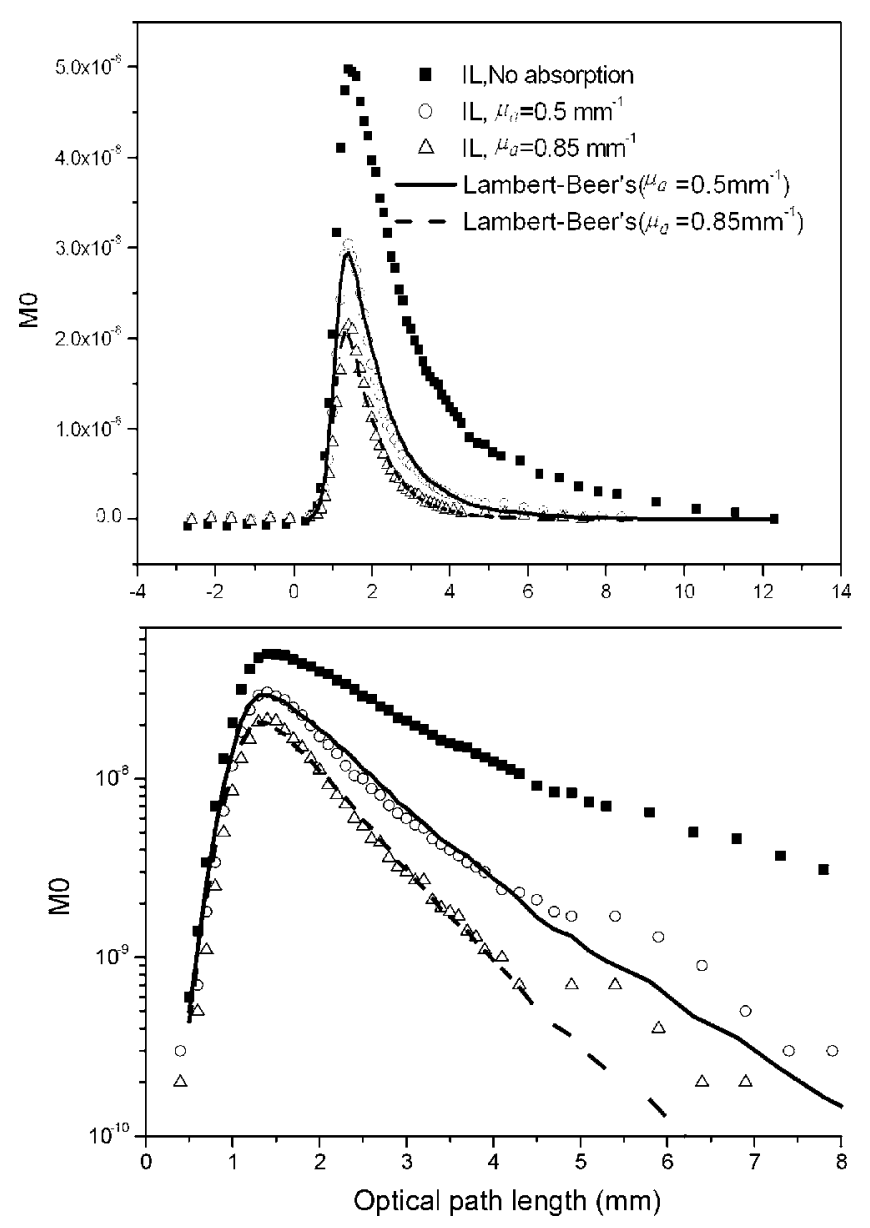

Fig. 4 Optical path length distributions estimated from the zero-order moment of the phase modulation peak for an aqueous Intralipid suspension $\left(\mu_{a}=0.001 \mathrm{~mm}^{-1}\right)$ and for identical suspensions with two different absorption coefficients $\left(0.50\right.$ and $\left.0.85 \mathrm{~mm}^{-1}\right)$, but equal reduced scattering coefficient (linear and logarithmic scales). The lines result from the application of Lambert-Beer's law on the experimental dataset for zero absorption.

ence arm, contains the ordinary homodyne signal due to the mutual interference of scattered light over almost equal optical path lengths in the sample. The power spectrum measured when the path length difference between the reference light and the scattered light is within the coherence length of light source, contains a heterodyne component at low frequencies, superimposed on the homodyne spectrum. This is due to the interference of scattered light with the component of the electric field of the reference light that is not phase modulated, as explained in the Introduction in Sec. 1, with reference to the phasor diagram shown in Fig. 1.

For the visco-elastic gel medium, no broadening of the line width is observed in Fig. 3. Unlike the dynamic media, there is no observable difference at low frequencies between the spectra for the three optical path lengths.

Estimations of path length distributions of photons in the aqueous Intralipid suspension $\left(\mu_{a}=0.001 \mathrm{~mm}^{-1}\right)$ and for identical suspensions with different absorption levels $(0.50$ and $0.85 \mathrm{~mm}^{-1}$ ) are shown in Fig. 4. The estimation of the optical path length distribution is obtained for increasing absorption levels. The minimum path length is the same for all 


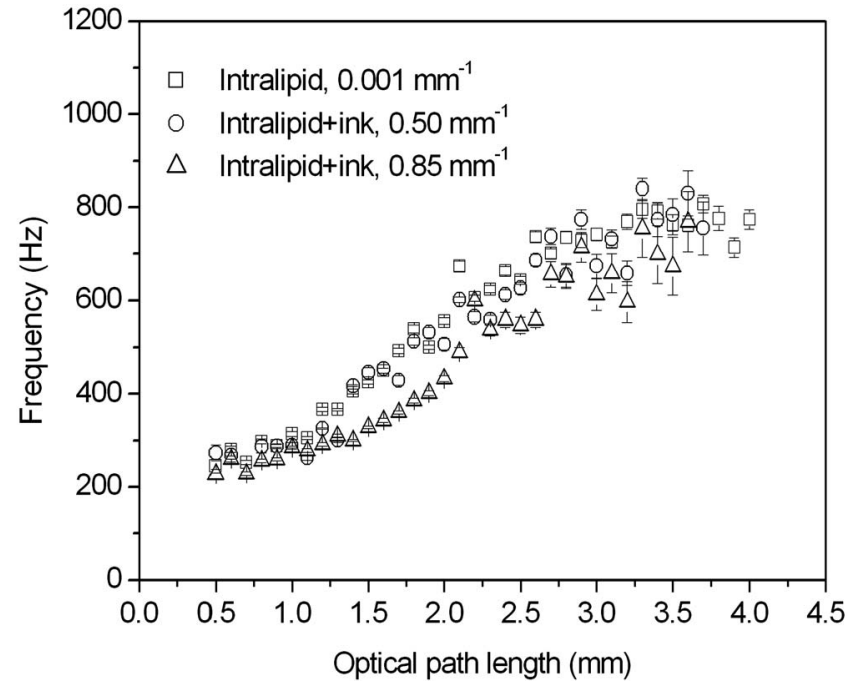

Fig. 5 The average Doppler shift extracted from the phase modulation peak as a function of the optical path length for an aqueous Intralipid suspension $\left(\mu_{a}=0.001 \mathrm{~mm}^{-1}\right)$ and for identical suspensions with two different absorption coefficients $\left(0.50\right.$ and $\left.0.85 \mathrm{~mm}^{-1}\right)$.

absorptions and is related to the fiber distance of $500 \mu \mathrm{m}$. At this path length, all the distributions start to increase independent of the absorption. As the photons with longer path length have a greater probability to be absorbed in an increasingly absorbing medium, $M_{0}$ decreases with the absorption. Hence the path length distribution narrows and shows a decrease in the average intensity as the absorption coefficient increases. Lambert-Beer's law can describe the effect of absorption on the path length distribution. According to the law of LambertBeer, the light intensity $I_{0}$ in an absorbing medium decays exponentially as $I(L)=I_{0} \exp \left(-L \mu_{a} / n\right)$, with $L$ being the optical path length, and $\mu_{a}$ and $n$ the absorption coefficient and the refractive index of the medium. To validate that the results shown in Fig. 4 represent true optical path length distributions, we verify whether the path length distribution of the original Intralipid and the same suspensions with high absorption coefficients are mutually related by Lambert-Beer's law. The path length distributions of original Intralipid multiplied by the exponential decay function $\exp \left(-L \mu_{a} / n\right)$ and the experimental data are shown in Fig. 4 in linear and logarithmic scales. There is good agreement between the experimental data and the calculated values (for $n=1.33, \mu_{a}=0.50$, and $0.85 \mathrm{~mm}^{-1}$ ) on the basis of Lambert-Beer's law up to an optical path length of $4.5 \mathrm{~mm}$, which proves that path length distributions have been correctly measured.

The average Doppler shift, measured from the Lorentzian width of Doppler-broadened phase modulation interference peaks, is represented in Fig. 5 as a function of the optical path length. The average Doppler shift increases with the optical path length, which can be expected from the increase in the number of scattering events with the optical path length. Further, for a given optical path length, the Doppler broadening of the modulation peak is equal for the media with zero absorption and the lowest nonzero absorption coefficient $\left(\mu_{a}\right.$ $\left.=0.50 \mathrm{~mm}^{-1}\right)$. But in the case of the highest absorption level $\left(\mu_{a}=0.80 \mathrm{~mm}^{-1}\right)$, the Doppler broadening is equal to those of the other absorption levels for optical path lengths up to

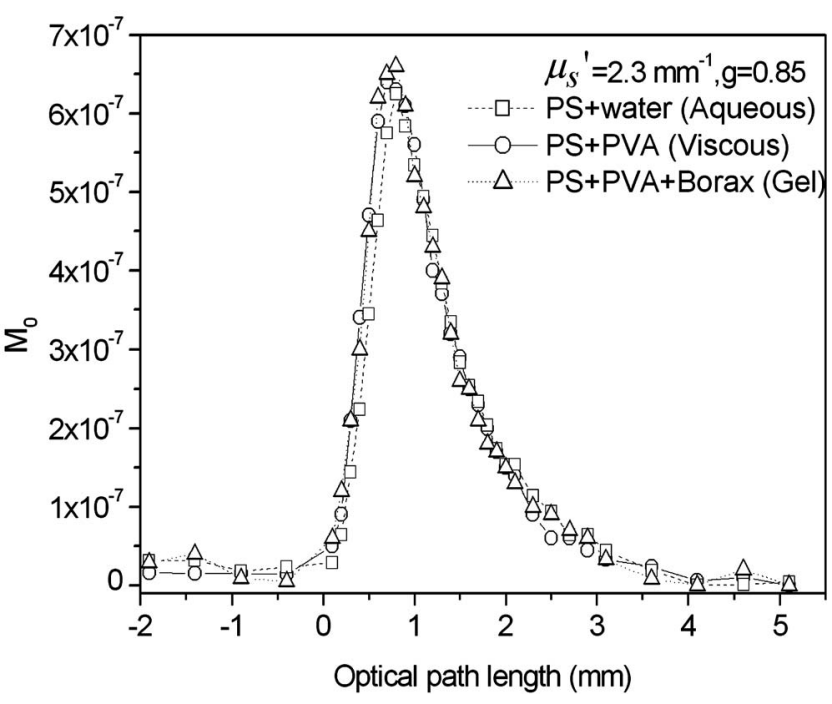

Fig. 6 Optical path length distributions estimated from the zero-order moment of the phase modulation peak, for viscous media (PVA +polystyrene microspheres), visco-elastic gel media (PVA +polystyrene microspheres+borax), and the aqueous suspension of polystyrene microspheres with different dynamical properties and identical optical properties $\left(\mu_{s}^{\prime}=2.35 \mathrm{~mm}^{-1}, g=0.85\right)$.

$1.1 \mathrm{~mm}$, while for large optical path lengths, the Doppler broadening is found to be decreased, in particular in the optical path length interval between 1.1 and $2 \mathrm{~mm}$.

The optical path length distributions measured for the viscous medium, the visco-elastic gel media, and the aqueous suspension of polystyrene microspheres with different dynamical properties and identical optical properties $\left(\mu_{s}^{\prime}\right.$ $=2.35 \mathrm{~mm}^{-1}, g=0.85$ ) are shown in Fig. 6 .

The average Doppler shifts observed for these media are represented in Fig. 7 as a function of the optical path length. For the PVA-PS viscous mixture, a decrease in the average Doppler shift is observed compared to the water suspension of polystyrene microspheres with identical optical properties. When borax was added to this PVA-PS mixture, an almost static gel was formed due to cross-linking, and the Lorentzian line width was further reduced for a given optical path length.

\section{Discussion}

We have presented optical path length distributions and pathlength-resolved Doppler shifts of multiply scattered light, extracted from the spectral peak that was generated by phase modulation of the reference arm in a low-coherence MachZehnder interferometer. As such, these data can also be obtained without modulation, but then we only can obtain information about photons that were Doppler shifted by the medium. Hence, phase modulation will enable us to measure path length distributions of static, mixed static, and dynamic media. A second advantage of using phase modulation is that the information can be shifted to higher frequencies, where often the noise level is lower and its spectrum is more flat than for low frequencies (Fig. 3). Without phase modulation, path length and Doppler broadening information must be retrieved from differences between the homodyne spectrum of photocurrent fluctuations generated by mutual interference of 
Varghese et al.: Path-length-resolved measurements...

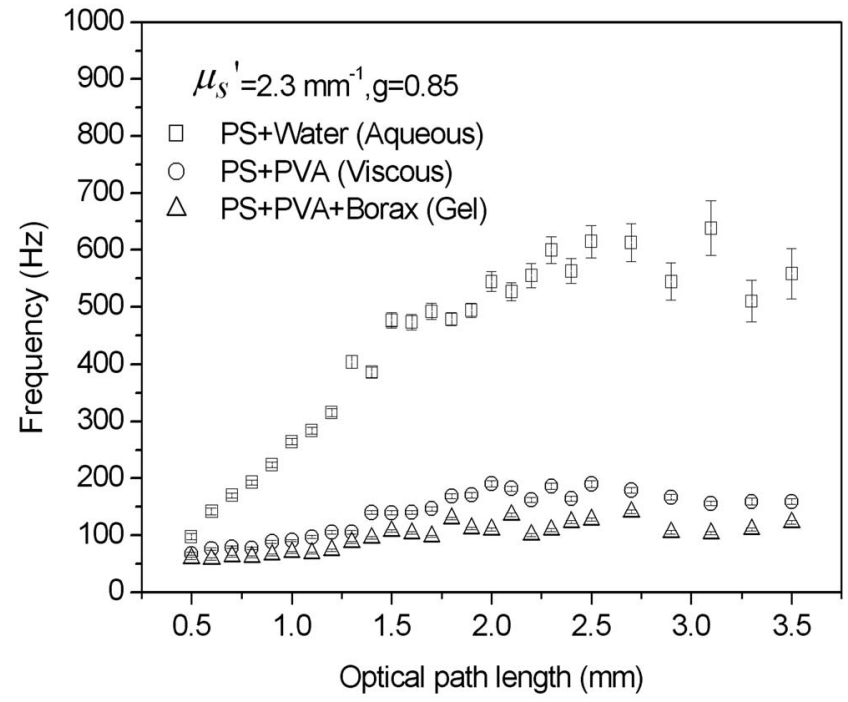

Fig. 7 The average Doppler shift extracted from the phase modulation peak as a function of the optical path length for viscous media (PVA +polystyrene microspheres), visco-elastic gel media (PVA +polystyrene microspheres+borax), and the aqueous suspension of polystyrene microspheres with different dynamical properties and identical optical properties $\left(\mu_{s}^{\prime}=2.35 \mathrm{~mm}^{-1}, g=0.85\right)$.

light through the sample, and the spectrum caused by interference of all detected light, which consists of a homodyne and a heterodyne part. Thus, in this case the heterodyne spectrum will occupy the same frequency range as the homodyne spectrum and the homodyne fluctuations plus real noise will act as noise to the heterodyne spectrum, which contains the path length information. As can be seen in Fig. 3, with phase modulation the heterodyne spectrum is shifted to a frequency range where the power spectral level of the homodyne fluctuations plus noise is more than 1 order of magnitude lower, while the level of the noise is a factor 2.5 lower. When the signal-to-noise ratio is expressed as the zero-order moment $M_{0}$ of the total spectrum for the retroreflector position where maximum interference is measured, divided by the value of $M_{0}$ at a position where no interference can be expected, it appears that phase modulation enhances the signal-to-noise ratio by a factor of approximately 30 .

The effect of intermodal dispersion of multimode fibers on the path length resolution is reduced by the choice of gradedindex fibers, with the upper limit of multimode dispersion, which is still of the order of $1 \mathrm{~mm} / \mathrm{m}$. This latter number suggests that for the fiber length in our setup, dispersion could have a considerable influence on the measured path length distributions. We measured the path length resolution of our setup by replacing the sample arm by a mirror, and the position of the retroreflector was translated for which a heterodyne signal was obtained. The path length resolution measured was $50 \mu \mathrm{m}$, and this degradation in resolution from $18 \mu \mathrm{m}$ (as expected from the coherence length of the light source) was due to the modal dispersion of multimode graded index detection fibers. This resolution of $50 \mu \mathrm{m}$ is sufficient for measuring path length distributions of $10 \mathrm{~mm}$ and thus do not suggest a significant deteriorating effect of intermodal dispersion in the path-length-resolved measurements. The results presented here exhibit the trends that must be expected for path length distributions and Doppler shifts of multiple scattered light. Optical path length distributions spanning a range from 0 to $11 \mathrm{~mm}$ are measured. Depending on the scattering properties of the medium, optical path length distributions exceeding $12 \mathrm{~mm}$ can also be measured for media with a lower absorption coefficient. It might be useful, but it is not necessary, to normalize the measured optical path lengths by the mean free path. For an increasing absorption coefficient, the estimated path length distributions narrow and show a decrease in the average intensity. This mutual relationship between the path length distributions obtained for various absorption levels through Lambert-Beer's law proves the correctness of these results.

The observed line width broadening results from the detection of multiple scattered photons, and as the number of scattering events increases, the width of the peak increases with optical path length. For a given medium with a constant scattering coefficient but absorption coefficients $\mu_{a}=0.001$ and $0.50 \mathrm{~mm}^{-1}$, the Doppler broadening of path-lengthresolved heterodyne spectra is shown to be independent of the absorption level for a given optical path length. But in the case of a higher absorption coefficient $\left(\mu_{a}=0.85 \mathrm{~mm}^{-1}\right)$, for optical path lengths between 1.3 and $2 \mathrm{~mm}$, the Doppler broadening deviates from those obtained for the lower absorption levels. This absorption level would correspond to an unrealistically high relative blood volume of $45 \%$. The nonzero absorption levels used in this study are larger than the values found on average in normally perfused tissue. Therefore, our results indicate that for absorption levels realistic for tissue, our method enables Doppler measurements independent of the absorption level of the medium in which the moving particles are embedded. A similar behavior may be obtained for the effect of the scattering coefficient of a static tissue matrix with moving particles.

The path length distributions measured for viscous media, visco-elastic gel media, and the aqueous suspension of polystyrene microspheres $\left(\mu_{s}^{\prime}=2.35 \mathrm{~mm}^{-1}, g=0.85\right)$ are identical, and the variation of the dc value for all previous three samples is within $7 \%$. Thus, independent of the Brownian motion of the scatterers, our method can measure realistic optical path length distributions. For a given optical path length, the average Doppler shift is reduced in the case of the viscous waterPVA-PS mixture, compared to the corresponding water suspension of polystyrene microspheres. In the case of gel, the scatterers can make restricted Brownian motion about their average position, and the effect of Doppler broadening due to multiple scattering events is further suppressed. We used an average of 1000 spectra to obtain path length distributions from dynamic samples. However, in the case of a gel medium, an average of 5000 spectra was needed to measure a decent path length distribution. This observation that averaging over a long time was needed in a static medium is similar to the observation by Tualle, Tinet, and Avrillier, ${ }^{9}$ that the path length distributions of multiple scattered light transmitted to a solid scattering medium showed a random shape, and they used an ensemble averaging of measurements obtained at 600 positions on the scattering medium. In our visco-elastic gel medium, the slow motion of the scattering particles allowed for averaging of multiple configurations of the medium, which is equivalent to spatial averaging. The background of 
the apparent inherent noisiness of path length distributions in static media as compared to dynamic media is not clear.

The experimental results presented here are performed on nonflowing samples, in which the Doppler shift was imparted due to Brownian motion of particles. The common method of calibration used in conventional laser Doppler perfusion instruments is based on the Brownian motion of latex spheres in water solution. The calibration signal of these instruments resulting from Brownian motion is within the physiological range. Also, laser Doppler perfusion monitors (Perimed PF5000 and Moorlab) measured similar amounts of Doppler broadening in the motility standard (an aqueous suspension of polystyrene microspheres with a diameter of $320 \mathrm{~nm}$ ) and in vivo on the cortex of a pig's kidney. ${ }^{14}$ Our preliminary measurements on a flowing sample (a rubber tube filled with an aqueous suspension of $20 \%$ of Intralipid, $20 \%$ which was pumped by a peristaltic pump for different flow velocities) indicate that this method has sufficient dynamic range in measuring Doppler shifts from flowing samples. Over a range of velocities $(0,3.68$, and $5.15 \mathrm{~mm} / \mathrm{s})$, the Doppler shift per path length bears a linear relation with the velocity for a range of optical path lengths from 0.4 to $1.4 \mathrm{~mm}$. The average Doppler shift per unit path length measured was 219,843 , and $1006 \mathrm{~Hz}$ for flow velocities of $0,3.68$, and $5.15 \mathrm{~mm} / \mathrm{s}$, respectively. Also, for a given velocity, the Doppler shift increases with optical path length due to the relative increase in the number of multiple scattering events in the sample arm.

Future experiments on well-defined and calibrated samples of polystyrene microspheres with different scattering levels and anisotropies, which are more typical for biological tissue, will reveal the accuracy of our method for Doppler spectra, and the results will be compared with the predictions of diffusive wave spectroscopy. With the experimental approach presented here, the dynamic properties of turbid media can be measured independent of optical properties, which is an important condition for absolute perfusion measurements for various tissue types. We aim to extend this method to pathlength-sensitive measurement of tissue perfusion. Furthermore, since for longer optical path lengths the slope of the path length distributions is affected by absorption, differences in path length distribution for various blood concentrations in skin may reveal the hemoglobin content of skin. Determination of blood oxygenation may be an important application of spectroscopic implementation of our technique.

\section{Conclusion}

We develop an improved method to determine path length distributions of multiple scattered light in static and dynamic turbid media using phase-modulated coherence-gated interferometry. Optical path length distributions are measured with optical path length ranges between 0 and $11 \mathrm{~mm}$. By relating experimental estimations of optical path length distributions obtained for various absorption coefficients by LambertBeer's law, we prove to be able to measure path length distributions of multiple scattered photons in turbid media. We have shown that path-length-resolved dynamic light scattering can measure the dynamic properties of a medium, indepen- dent of its optical absorption properties, at least when absorption levels are applied in the range found for biological tissues.

Using the fiber optic Mach-Zehnder interferometer with phase modulation enables us to obtain path length distributions and to extract path-length-resolved information from mixed static and dynamic turbid media. By its noninvasive nature in measuring path-length-resolved dynamic light scattering, the method has potential applications in the fields of fundamental as well as applied research in monitoring the spatial and temporal variations in optical properties in turbid media, blood perfusion in tissue, and its hemoglobin content.

\section{Acknowledgments}

This work was sponsored by the Netherlands Technology Foundation STW (grant TTF 5840). Johan van Hespen and Anton Hollink are acknowledged for technical support.

\section{References}

1. A. P. Shepherd and P. Å. Öberg, Laser-Doppler Blood Flowmetry, Kluwer Academic, Boston, MA (1990).

2. B. Chance, J. S. Leigh, H. Miyake, D. S. Smith, S. Nioka, R. Greenfeld, M. Finander, K. Kaufmann, W. Levy, M. Young, P. Cohen, H. Yoshioka, and R. Boretsky, "Comparison of time-resolved and unresolved measurements of deoxyhemoglobin in brain," Proc. Natl. Acad. Sci. U.S.A. 85, 4971-4975 (1988).

3. E. Gratton and M. Limkerman, "A continuously variable frequency cross-correlation phase fluorometer with picosecond resolution," Biophys. J. 44, 315-324 (1983).

4. A. Wax, C. Yang, R. R. Dasari, and M. S. Feld, "Path-length-resolved dynamic light scattering: Modeling the transition from single to diffusive scattering," Appl. Opt. 40, 4222-4227 (2001).

5. G. Popescu and A. Dogariu, "Optical path-length spectroscopy of wave propagation in random media," Opt. Lett. 24, 442 (1999).

6. K. K. Bizheva, A. M. Siegel, and D. A. Boas, "Path-length-resolved dynamic light scattering in highly scattering random media: the transition to diffusing wave spectroscopy," Phys. Rev. E 58, 7664-7667 (1998).

7. A. L. Petoukhova, W. Steenbergen, and F. F. M. de Mul, "Path-length distribution and path-length resolved Doppler measurements of multiply scattered photons by use of low-coherence interferometer," Opt. Lett. 26, 1492-1494 (2001).

8. A. L. Petoukhova, W. Steenbergen, T. G. van Leeuwen, and F. F. M. de Mul, "Effects of absorption on coherence domain path length resolved dynamic light scattering in the diffuse regime," Appl. Phys. Lett. 81, 595-597 (2002).

9. J. M. Tualle, E. Tinet, and S. Avrillier, "A new and easy way to perform time resolved measurements of the light scattered by turbid medium," Opt. Commun. 189, 211-220 (2001).

10. D. Huang, E. A. Swanson, C. P. Lin, J. S. Schuman, W. G. Stinson, W. Chang, M. R. Hee, T. Flotte, K. Gregory, C. A. Puliafito, and J. G. Fujimoto, "Optical coherence tomography," Science 254, 1178 (1991).

11. K. Ishii, R. Yoshida, and T. Iwai, "Single-scattering spectroscopy for extremely dense colloidal suspensions by use of a low-coherence interferometer," Opt. Lett. 30, 555-557 (2005).

12. G. E. Nilsson, "Signal processor for laser Doppler tissue flowmeters," Med. Biol. Eng. Comput. 22, 343-348 (1984).

13. S. T. Flock, S. L. Jacques, B. C. Wilson, W. M. Star, and M. J. C. van Gemert, "Optical properties of intralipid: a phantom medium for light propagation studies," Lasers Surg. Med. 12, 510-519 (1992).

14. A. L. Petoukhova, W. Steenbergen, F. Morales, R. Graaff, E. D. de Jong, J. M. Elstrodt, F. F. M. de Mul, and G. Rakhorst, "Instrument independent flux units for laser Doppler perfusion monitoring in a multi-device study on the renal cortex," Microvasc. Res. 66, 83-90 (2003) 